\title{
Rotating Models of Low Mass Giants: Rotational Evolution and Surface Abundance Anomalies
}

\author{
Julio Chanamé, Marc Pinsonneault, and Don Terndrup \\ The Ohio State University, $140 \mathrm{~W}$. 18th Ave., Columbus, $\mathrm{OH} 43210$
}

\begin{abstract}
.
We present some first results of stellar models of low mass, solar metallicity giants that include rotation and the mixing associated with it. We base our choice of initial conditions and input physics on observational constraints obtained for main sequence (MS) and horizontal branch (HB) stars, and obtain what can be regarded as a "best case scenario" for rotational mixing. These observations suggest local conservation of angular momentum in the cores and constant specific angular momentum in convective regions on the red giant branch (RGB). We show that under these circumstances and with moderate rotation rates at the turnoff it is possible to obtain enough mixing in order to reproduce the anomalous abundance ratios of the CNO species seen in giants. It is also shown that solid body rotation in the convective envelope fails to account for the observations. It is found that rotational mixing is inefficient on the lower RGB, so there is no need to invoke any inhibiting role of $\mu$-gradients.
\end{abstract}

\section{Initial Conditions and Rotational Evolution}

Internal mixing determines the evolution of the measured surface abundances. The amount of rotational mixing depends on the angular momentum distribution throughout the star. Therefore, observational constraints on the distribution of angular momentum are key ingredients for understanding rotational mixing.

Observations of rotation rates of HB stars in globular clusters (Peterson et al. 1995) indicate that a high fraction of the MS angular momentum content survives the extensive mass loss experienced during the giant branch, as found by Sills \& Pinsonneault (2000). Therefore, in our models we require local conservation of angular momentum in radiative regions, and enforce constant specific angular momentum $(\mathrm{J} / \mathrm{M})$ in convective regions during the RGB. We contrast these assumptions against solid body rotation in the convective envelope. A starting solid body profile is assigned at the turnoff. Mass loss is not included in the results presented here.

The important difference between the two rotation laws in convective regions is that they concentrate the angular momentum content in different regions of the star. In the case of constant specific angular momentum the bulk of the angular momentum is concentrated where the mass is, namely, the radiative interior. Solid body rotation, on the other hand, concentrates most of the star's angular momentum in the outer layers $\left(\mathrm{J} / \mathrm{M} \propto \Omega \mathrm{r}^{2}\right)$. 

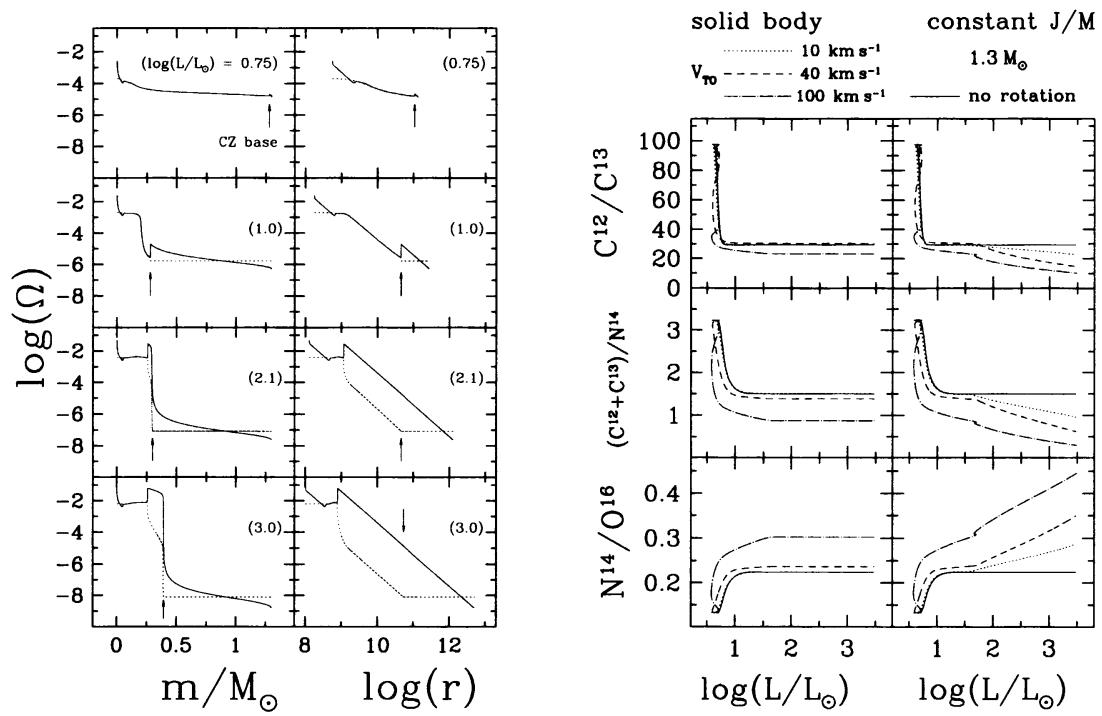

Figure 1. Left. Evolution of the angular velocity profile for a star of $1.3 \mathrm{M}_{\odot}$ and $40 \mathrm{~km} / \mathrm{s}$ at turnoff. Solid lines represent constant $\mathrm{J} / \mathrm{M}$, and dotted lines represent solid body rotation. Right. Evolution of the CNO surface abundance ratios for two different rotation laws enforced in the convective envelope, showing the dependence on the rotational velocity at turnoff for a $1.3 \mathrm{M}_{\odot}$ star.

\section{Mixing - Evolution of the Surface Abundances}

We show the evolution of the surface abundances in the right plot of Figure 1, where the standard first dredge-up is illustrated as the solid lines. The ${ }^{12} \mathrm{C} /{ }^{13} \mathrm{C}$ ratio drops from the initial (solar) value of $\approx 90$ to $\approx 25$, while the middle and bottom panels show the characteristic overall net conversion of carbon and oxygen into nitrogen, typical of CNO processing in the hot interior of the star.

Rotating models mix more than the standard one, and the most favorable situation for mixing is that with constant $\mathrm{J} / \mathrm{M}$ enforced in convective regions. This is the same condition required to explain the data on HB rotation rates.

Our models do not include any inhibiting role of $\mu$-gradients, so an important conclusion follows: although at work, rotational mixing is inefficient on the lower RGB, regardless of the presence of $\mu$-barriers and any inhibiting effect these might have on mixing. Later, the mixing becomes increasingly important only after the RGB bump $\left(\log \left(\mathrm{L} / \mathrm{L}_{\odot}\right) \approx 1.6\right)$, when the star's luminosity, and so the energy available for rotational mixing, is about an order of magnitude larger than at the subgiant branch.

\section{References}

Peterson, R.C., Rood, R.T., \& Crocker, D.A. 1995, ApJ 453, 214

Sills, A. \& Pinsonneault, M. 2000, ApJ 540, 489 